\title{
Internal mammary lymph node management - further direction
}

This article was published in the following Dove Press journal:

OncoTargets and Therapy

21 February 2017

Number of times this article has been viewed

D Vrana
J Gatek

'Department of Oncology, ${ }^{2}$ Institute of Molecular and Translational Medicine, Faculty of Medicine and Dentistry, Palacky University, Olomouc, ${ }^{3}$ Department of Surgery, Atlas Hospital, ${ }^{4}$ Faculty of Humanities, Tomas Bata University in Zlín, Zlín, Czech Republic
Correspondence: D Vrana Department of Oncology, Faculty of Medicine and Dentistry, Palacky University, I.P. Pavlova 6, Olomouc, Czech Republic

Tel +420 588444295

Email davvrana@gmail.com

\section{Dear editor}

We read the article titled "Internal mammary sentinel lymph node biopsy: abandon or persist?" by Qiu et al with high interest. This was an excellent paper regarding the contemporary management of internal mammary lymph nodes (IMLN) in early-stage breast cancer ${ }^{1}$ and we would like to take this opportunity to comment on this paper.

There are several unresolved questions regarding early-stage breast management including axillary staging, clear resection margin, or IMLN..$^{2-4} \mathrm{We}$ have been focusing on the issues of IMLN for almost a decade and just recently published our data regarding IMLN management. We absolutely agree that one has to carefully balance the benefit and potential risks of biopsy or radiotherapy of IMLN. Our current practice is not to do biopsy/not to irradiate IMLN unless they are clinically enlarged. However, we are always cautious about the visualization of sentinel lymph nodes by sentinel lymph node biopsy (SLNB) in internal mammary chain. We reviewed all breast cancer patients who underwent SLNB in our department from 2008 to 2012 to achieve 5 years median follow-up and to figure out whether IMLN drainage acts as a negative prognostic factor in case if they are left without any management. Our trial included more than 700 patients and the results obtained concluded no detrimental effect of IMLN drainage during SLNB procedure. There was no statistically significant difference in overall survival and local or distant recurrence rate. ${ }^{5}$ Our findings are in contrast to that of other trials ${ }^{6}$ recommending radiotherapy for IMLN; even trials such as MA.20 or EORTC 22922 that focused on axillary lymph node radiotherapy have recommended radiotherapy for IMLN. However, we believe that the difference observed might be due to the difference in the enrollment period. Our trial enrolled patients in the era of targeted therapy, taxanes, and dose-dense chemotherapy as a standard for breast cancer patients. These chemotherapy regimens may potentially compensate for uncertainties in the local management of IMLN. We believe these results are in line with the generally accepted contemporary trend toward less-radical locoregional treatment of the early-stage breast cancer, and further trials have to be carried out to confirm our findings.

\section{Disclosure}

The authors report no conflicts of interest in this communication. 


\section{References}

1. Qiu PF, Liu YB, Wang YS. Internal mammary sentinel lymph node biopsy: abandon or persist? Onco Targets Ther. 2016;9:3879-3882.

2. Vrana D, Gatek J, Cwiertka K, Lukesova L, Koranda P. Internal mammary node management in breast cancer. A review. Biomed Pap Med Fac Univ Palacky Olomouc Czech Repub. 2013;157(3):261-265.

3. Gatek J, Vrana D, Melichar B, et al. Significance of the resection margin and risk factors for close or positive resection margin in patients undergoing breast-conserving surgery. $J$ BUON. 2012;17(3):452-456.
4. Gatek J, Vrana D, Hnatek L, et al. Sentinel node biopsy and neoadjuvant chemotherapy in the treatment of breast cancer. $J$ BUON. 2012;17(2):265-270.

5. Lukesova L, Vrana D, Svach I, et al. B. Prognostic influence of internal mammary node drainage in patients with early-stage breast cancer. Anticancer Res. 2016;36(12):6641-6646.

6. Thorsen LB, Offersen BV, Danø H, et al. DBCG-IMN: a populationbased cohort study on the effect of internal mammary node irradiation in early node-positive breast cancer. J Clin Oncol. 2016;34(4):314-320. 


\section{Authors' reply \\ Peng-Fei Qiu \\ Yong-Sheng Wang}

Breast Cancer Center, Shandong Cancer Hospital Affiliated to Shandong University, Jinan, People's Republic of China

Correspondence: Yong-Sheng Wang

Breast Cancer Center, Shandong Cancer Hospital Affiliated to Shandong University, 440 Jiyan Road, Jinan, Shandong 250I I7, People's Republic

of China

Tel +86531 67626211

Email wangysh2008@aliyun.com

\section{Dear editor}

We appreciate the letter from Professors Vrana and Gatek regarding our article titled "Internal mammary sentinel lymph node biopsy: abandon or persist?". ${ }^{1}$ We have been following their publications regarding internal mammary lymph nodes (IMLN) management since the publication of their article titled "Prognostic influence of internal mammary node drainage in patients with early-stage breast cancer" in December $2016^{2}$ and we share their interest on this topic.

Their trials retrospectively reviewed patients with breast cancer who underwent sentinel lymph node biopsy (SLNB), and IMLN drainage was assessed as a potential risk factor for local and distant disease recurrences. We agree that the drainage of IMLN is unlikely to have a detrimental effect on patient outcome. However, their results did not mean that IMLN does not need any management, because presence of IMLN drainage should not be interpreted as having IMLN metastasis and absence of IMLN drainage as not having IMLN metastasis. We believe that the assessment of prognostic influence should be based on IMLN metastasis rather than only on IMLN drainage. Moreover, their study population had been limited to the clinically axilla lymph node (ALN)negative patients, and the incidence of IMLN metastasis in these patients is only about $10 \%$ as reported. ${ }^{3}$ Therefore, the difference was small even if there was a difference between the patients with and without IMLN drainage.

It was recognized that the benefit of systemic therapy on locoregional control had transformed the pattern of breast cancer therapy, and within the changing treatment approach - more systemic therapy, less locoregional therapy - clinicians should deliberate the application of regional IMLN therapy. The absolute benefit of internal mammary radiotherapy (IMRT) has been decreased with the applications of effective systemic treatments, but it still exists. ${ }^{4}$ The 2016 NCCN Guidelines recommend IMRT for patients with MLN therapy. The absolute be and strongly consider IMRT for patients with 1-3 positive ALN (category 2A). Studies of extended radical mastectomy reported that 36.8\%-46.2\% patients with $\geq 4$ positive ALN and $18.8 \%-26.7 \%$ patients with 1-3 positive ALN identified with IMLN metastases. ${ }^{5,6}$ Therefore, IMRT should be tailored and balanced between the benefit and potential risks, and internal mammary sentinel lymph node biopsy (IM-SLNB) might be a minimally invasive staging technique that guides the tailored IMRT. In our recent study, we tried injecting radiotracer with a modified technique (periareolar intraparenchymal, high volume and ultrasound guidance) and obtained a high IM-SLN detection rate of $75.1 \%$. We found that the IM-SLN metastasis rate was only $8.1 \%$ in clinically ALN-negative patients whereas it was $20.5 \%$ in clinically ALN-positive patients, and individual IMRT strategy could be guided based on IM-SLNB results. ${ }^{7-10}$ We recommend that IM-SLNB should be performed in all clinically ALN-positive patients and selectively in high-risk (upper inner quadrant tumor and/or ALN-positive) clinically ALN-negative patients.

Finally, we encourage that IM-SLNB study should still be performed in breast cancer patients, especially for high-risk IMLN involvement, and prognostic assessment of IM-SLNB metastasis is essential in order to improve diagnosis and provide a more individual IMRT and a more accurate prognosis.

\section{Disclosure}

The authors report no conflicts of interest in this communication.

\section{References}

1. Qiu PF, Liu YB, Wang YS. Internal mammary sentinel lymph node biopsy: abandon or persist? Onco Targets Ther. 2016;9:3879-3882.

2. Lukesova L, Vrana D, Svach I, et al. Prognostic influence of internal mammary node drainage in patients with early-stage breast cancer. Anticancer Res. 2016;36(12):6641-6646.

3. Chen RC, Lin NU, Golshan M, Harris JR, Bellon JR. Internal mammary nodes in breast cancer: diagnosis and implications for patient management - a systematic review. J Clin Oncol. 2008;26(30):4981-4989.

4. Thorsen LB, Offersen BV, Danø H, et al. DBCG-IMN: a populationbased cohort study on the effect of internal mammary node irradiation in early node-positive breast cancer. J Clin Oncol. 2016;34(4):314-320.

5. Veronesi U, Marubini E, Mariani L, Valagussa P, Zucali R. The dissection of internal mammary nodes does not improve the survival of breast cancer patients. 30-year results of a randomised trial. Eur J Cancer. 1999;35(9):1320-1325.

6. Huang O, Wang L, Shen K, et al. Breast cancer subpopulation with high risk of internal mammary lymph nodes metastasis: analysis of 2,269 Chinese breast cancer patients treated with extended radical mastectomy. Breast Cancer Res Treat. 2008;107(3):379-387.

7. Qiu PF, Liu JJ, Liu YB, Yang GR, Sun X, Wang YS. A modified technology could significantly improve the visualization rate of the internal mammary sentinel lymph nodes in breast cancer patients. Breast Cancer Res Treat. 2012;136(1):319-321.

8. Qiu PF, Zhao RR, Liu YB, Wang YS. Internal mammary sentinel lymph node biopsy should still be performed, especially in the patient with clinically positive axillary lymph nodes. Breast. 2013;22(5):999-1000. 
9. Cong BB, Qiu PF, Wang YS. Internal mammary sentinel lymph node biopsy: minimally invasive staging and tailored internal mammary radiotherapy. Ann Surg Oncol. 2014;21(7):2119-2121.
10. Qiu PF, Cong BB, Zhao RR, et al. Internal mammary sentinel lymph node biopsy with modified injection technique: high visualization rate and accurate ataging. Medicine. 2015;94(41):e1790.

Dove Medical Press encourages responsible, free and frank academic debate. The content of the OncoTargets and Therapy 'letters to the editor' section does not necessarily represent the views of Dove Medical Press, its officers, agents, employees, related entities or the OncoTargets and Therapy editors. While all reasonable steps have been taken to confirm the content of each letter, Dove Medical Press accepts no liability in respect of the content of any letter, nor is it responsible for the content and accuracy of any letter to the editor.

\section{Publish your work in this journal}

OncoTargets and Therapy is an international, peer-reviewed, open access journal focusing on the pathological basis of all cancers, potential targets for therapy and treatment protocols employed to improve the management of cancer patients. The journal also focuses on the impact of management programs and new therapeutic agents and protocols on

\section{Dovepress}

patient perspectives such as quality of life, adherence and satisfaction. The manuscript management system is completely online and includes a very quick and fair peer-review system, which is all easy to use. Visit http://www.dovepress.com/testimonials.php to read real quotes from published authors.

Submit your manuscript here: http://www.dovepress.com/oncotargets-and-therapy-journal 